\title{
Endocannabinoid system and the expression of endogenous ceramides in human hepatocellular carcinoma
}

\author{
JIAYONG YANG ${ }^{1,2}$, YIFENG TIAN ${ }^{3,4}$, RUIHE ZHENG ${ }^{5}$, LEI LI ${ }^{5}$ and FUNAN QIU ${ }^{3,4}$ \\ ${ }^{1}$ Department of Pharmacy, The Fifth Hospital of Xiamen, Xiamen, Fujian 361101; ${ }^{2}$ Department of Pharmacy, \\ The First Affiliated Hospital of Xiamen University, Xiamen, Fujian 361003; ${ }^{3}$ Provincial Clinical College, \\ Fujian Medical University, Fuzhou, Fujian 350004; ${ }^{4}$ Department of Hepatobiliary Surgery, \\ Fujian Provincial Hospital, Fuzhou, Fujian 350001; ${ }^{5}$ Eye Institute of Xiamen University, \\ School of Medicine, Xiamen University, Xiamen, Fujian 361102, P.R. China
}

Received May 3, 2018; Accepted April 15, 2019

DOI: $10.3892 / \mathrm{ol} .2019 .10399$

\begin{abstract}
The endogenous lipid metabolism network is associated with the occurrence and progression of malignancies. Endocannabinoids and ceramides have demonstrated their anti-proliferative and pro-apoptotic properties in a series of cancer studies. The aim of the present study was to evaluate the expression patterns of endocannabinoids and endogenous ceramides in 67 pairs of human hepatocellular carcinoma (HCC) tissues and non-cancerous counterpart controls. Anandamide (AEA), the major endocannabinoid, was reduced in tumor tissues, probably due to the high expression and activity of fatty acid amide hydrolase. Another important endocannabinoid, 2-arachidonylglycerol (2-AG), was elevated in tumor tissues compared with non-tumor controls, indicating that the biosynthesis of 2-AG is faster than the degradation of 2-AG in tumor cells. Furthermore, western blot analysis demonstrated that cannabinoid receptor 1 was downregulated, while cannabinoid receptor 2 was elevated in HCC tissues, in accordance with the alterations in the levels of AEA and 2-AG, respectively. For HCC tissues, the expression levels of C18:0, 20:0 and 24:0-ceramides decreased significantly, whereas C12:0, 16:0, 18:1 and 24:1-ceramides were upregulated, which may be associated with cannabinoid receptor activation and stearoyl-CoA desaturase protein downregulation. The exact role of endocannabinoids and ceramides in regulating the fate of HCC cells requires further investigation.
\end{abstract}

Correspondence to: Mr. Jiayong Yang, Department of Pharmacy, The Fifth Hospital of Xiamen, 101 Min'an Road, Xiamen, Fujian 361101, P.R. China

E-mail: yjy_158@163.com

Mr. Funan Qiu, Provincial Clinical College, Fujian Medical University, 88 Jiaotong Road, Fuzhou, Fujian 350004, P.R. China

E-mail: qiufunan@medmail.com.cn

Key words: endocannabinoids, cannabinoid receptors, ceramides, hepatocellular carcinoma

\section{Introduction}

Primary hepatocellular carcinoma (HCC) remains the third leading cause of cancer-associated mortality in China. Clinical treatment modalities include surgical resection, transplantation, chemotherapy and radiotherapy. The 5-year survival rate of patients with HCC was 5-30\% between 2000 and 2014 (1). The main reason is that the initial symptoms of $\mathrm{HCC}$ are occult, and the majority of patients are diagnosed only at an advanced stage. Therefore, there is an urgent need to identify early HCC biomarkers and novel treatment targets.

In recent years, the reprogramming of metabolic pathways in tumor cells is a field of cancer research that has attracted attention $(2,3)$. De novo lipogenesis increases in liver cancer cells, and the endogenous lipid metabolism network changes significantly. Endocannabinoids are a class of endogenous lipids that target cannabinoid receptors 1 and $2\left(\mathrm{CB}_{1 / 2}\right)$, the most extensively investigated of which are anandamide (AEA) and 2-arachidonylglycerol (2-AG). Endocannabinoids modulate multiple cell survival-associated signaling pathways, including extracellular signal-regulated kinase (ERK) (4), p38 mitogen-activated protein kinase (MAPK) (5) and the ceramide pathways (6) in breast cancer (7), prostate cancer (8), rectal cancer (9) and glioma (10). Ceramides are widely distributed as sphingolipid messengers involved in apoptosis and cell cycle arrest. It was recently demonstrated that $\mathrm{CB}$ receptor activation-dependent apoptosis signaling is associated with ceramide accumulation (6).

Increased synthesis of endogenous mono-unsaturated fatty acids (MUFA) is another biochemical hallmark of cancer (11). Previous reports have shown that high expression of stearoyl-CoA desaturase-1 (SCD1) catalyzes the desaturation of saturated fatty acids (SFA), which provides abundant MUFA substrates for membrane biosynthesis during tumor cell proliferation. Inhibition of SCD1 suppressed the synthesis of MUFA and induced de novo synthesis of long-chain ceramides, which is a major mechanism mediating apoptosis in a variety of tumor cells (12).

The endogenous lipid metabolism network is associated with the occurrence and progression of malignancies. The abundance of prostaglandin E2 in the tumor microenvironment 
further impedes $\mathrm{T}$ cell infiltration and cancer immune evasion (13). However, the expression, metabolism and regulation of these lipids in HCC tissues have yet to be elucidated. In the present study, the pathological data from 67 patients with HCC who underwent surgical resection were retrospectively analyzed, and the differences in the levels of endogenous lipids and metabolic enzymes between tumor tissues and their non-tumor counterparts were evaluated. Examination of specific lipid metabolism profiles may provide targets and markers for HCC treatment and early diagnosis.

\section{Materials and methods}

Human tissue samples. Samples from cancer tissues and their adjacent normal counterparts were obtained from 67 patients who had undergone surgical resection for HCC at the Fifth Hospital of Xiamen (Xiamen, China) between January 2015 and December 2016. The mean age of the patients was $52.6 \pm 11.3$ years (range, $18-76$ years). In total, $83.6 \%$ of the patients were male, consistent with a prior report that HCC is more prevalent in men compared with women (14). The patients were diagnosed with HCC via pathological examination (data not shown). All patients were informed of the aims of this study, and they provided written informed consent for the investigation in accordance with the Ethics Committee in the Fifth Hospital of Xiamen, following the clinical registration guidelines in China. This study was approved by the Ethics Committee of the Fifth Hospital of Xiamen. Tumor-node-metastasis (TNM) stage was determined according to the World Health Organization TNM staging 7 th edition and the pathological analysis results. The samples were sectioned and some were stored at $-80^{\circ} \mathrm{C}$ for western blot and high-performance liquid chromatography (HPLC)-mass spectrometry (MS) analysis, whereas others were flash-frozen in liquid nitrogen for PCR analysis.

Reverse transcription-quantitative PCR (RT-qPCR) analysis. Total RNA was extracted from tissue samples using TRIzol reagent (Thermo Fisher Scientific, Inc.), and RNA concentration was measured with a spectrophotometer (Beckman Coulter, Inc.). Total RNA (1 $\mu \mathrm{g})$ was reverse-transcribed to cDNA with the ReverTra Ace ${ }^{\circledR}$ qPCR RT kit (Toyobo Life Science), according to the manufacturer's protocols, and amplified with Ex Taq DNA polymerase, according to the manufacturer's protocols. qPCR was carried out on an Applied Biosystems 7500 Fast Real-Time PCR System (Thermo Fisher Scientific, Inc.) with SYBR Premix Ex Taq II (Takara Bio Inc., Otsu, Japan). The thermocycling conditions were as follows: Denaturation at $95^{\circ} \mathrm{C}$ for $5 \mathrm{sec}$, annealing/extension at $60^{\circ} \mathrm{C}$ for $31 \mathrm{sec}$ (40 cycles). The quantitative values of mRNA were analyzed using the $2^{-\Delta \Delta \mathrm{Cq}}$ method (15) and normalized relative to the levels of $18 \mathrm{~S}$. Each sample was set up in triplicate and the experiments were repeated three times.

The primers were as follows: $N$-acylphosphatidylethanolamine-hydrolysingphospholipase D (NAPE-PLD), forward primer (F): 5'-TGGCTGGGACACGCG-3', reverse primer (R): 5'-GGGATCCGTGAGGAGGATG-3'; fatty acid amide hydrolase (FAAH), F: 5'-GCCTCAAGGAATGCTTCAGC-3', R: 5'-TGCCCTCATTCA GGCTCAAG-3'; monoacylglycerol lipase (MAGL), F: 5'-CATGTGGATTCCATGCAGAAAG-3',
R: 5'-AGGATTGGCAAGAACCAGAGG-3'; diacylglycerol lipase (DGL)- $\alpha$, F: 5'-AGAATGTCACCCTCGGAATGG-3', R: 5'-GTGGCTCTCAGCTTGACAAAGG-3'; CB 1 , F: 5'-AGC CTCTGGATAACAGCATGG-3', R: 5'-AATCTTGACCGT GCTCTTGATG-3'; CB 2 , F: 5'-CTCAGTGACCAGGTCAAG AAGG-3', R: 5'-TTTTGCCTCTGACCCAAGG-3'; ceramide synthases (Cer)S1, F: 5'-TTTGGCTCCCGCACAATGT-3', R: 5'-AAAAGCGAGATAGAGGTCCTCA-3'; CerS2, F: 5'-GCT CTTCCTCATCGTTCGATAC-3', R: 5'-GTGTAGCCACGT ACAGCTCA-3'; CerS3, F: 5'-CACCCAGCTGTCAAAGAG AAGG-3', R: 5'-AGGACGATATCCGAAAGGTGG-3'; CerS4, F: 5'-CCGGATCCCGTCCAGTTTCAACGAG-3', R: 5'-GG GAATTCGGCTATGTGGCTGTTGTG-3'; CerS5, F: 5'-GCTGCTCTTCGAGCGATTTAT-3', R: 5'-CCTCCGATG GCGAAACCAG-3'; CerS6, F: 5'-TTTGGCTCCCGCACA ATGT-3', R: 5'-AAAAGCGAGATAGAGGTCCTCA-3'; 18S, F: 5'-CAGCCACCCGAGATTGAGCA-3', R: 5'-TAGTAG CGACGGGCGGTGTG-3'.

Western blot analysis. Tumors and adjacent normal tissues were homogenized and sonicated $(50 / 60 \mathrm{~Hz})$ in ice-cold RIPA buffer (Beyotime Biotechnology, Jiangsu, China) for 10 times, $5 \mathrm{sec}$ each time. The suspension was centrifuged for $15 \mathrm{~min}$ at $4^{\circ} \mathrm{C}$ and $15,294 \mathrm{x}$ g. The total protein content was determined using a bicinchoninic acid protein assay kit (Thermo Fisher Scientific, Inc.), according to the manufacturer's protocols, with bovine serum albumin (BSA; Solarbio Science \& Technology Co. Ltd., Beijing, China) as the standard. Proteins $(20 \mu \mathrm{g})$ were mixed with sample buffer, boiled, and then separated by $15 \%$ SDS-PAGE. After transference to polyvinylidene difluoride membranes (GE Healthcare Life Sciences), the membrane was blocked in 5\% milk in Tris-buffered saline with $1 \%$ Tween-20 at room temperature for $1 \mathrm{~h}$, and incubated at $4^{\circ} \mathrm{C}$ overnight with primary antibodies against $\mathrm{CB}_{1}$ (Abcam; 1:1,000; cat. no. ab23703), $\mathrm{CB}_{2}$ (Abcam; 1:1,000; cat. no., ab3561), SCD1 (Abcam; 1:500; cat. no. ab19862) and $\beta$-actin (Sigma-Aldrich, Merck KGaA; 1:500; cat. no. A5316). The membranes were subsequently incubated with an HRP-conjugated anti-rabbit IgG antibody (Sigma-Aldrich, Merck KGaA; 1:10,000; cat. no. SAB5600127) for $1 \mathrm{~h}$ at room temperature. Protein bands were visualized using an Enhanced Chemiluminescence Plus kit (GE Healthcare Life Sciences). Quantitative analysis was performed using the ImageJ software (version 2.1.4.7; National Institute of Health) with $\beta$-actin as the endogenous control.

Endogenous lipid extraction and analysis. In total, $50 \mu \mathrm{g}$ frozen tissue samples were homogenized in $2 \mathrm{ml}$ methanol/ $\mathrm{H}_{2} \mathrm{O}$ mixture $(50: 50, \mathrm{v} / \mathrm{v})$ containing $\mathrm{C} 17: 1 \mathrm{FAE}$, and $\mathrm{C} 17: 0$ ceramide as internal standards. Endogenous lipids were extracted with $3 \mathrm{ml}$ chloroform, and then centrifuged for $10 \mathrm{~min}$ at $4^{\circ} \mathrm{C}$ and 3,000 $\mathrm{x}$ g. The organic phase was collected and dried under nitrogen by a nitrogen evaporator (Beijing TongTaiLian Technology Co., Ltd.). Lipids were re-dissolved in $1 \mathrm{ml}$ chloroform and transferred to small Silica Gel G columns. Endogenous FAEs and ceramides were eluted with methanol/chloroform (10:90, v/v), dried under nitrogen, reconstituted in $100 \mu \mathrm{l}$ methanol and detected using the $3200 \mathrm{Q}$ Trap HPLC-MS system (Applied Biosystems; Thermo Fisher Scientific, Inc.) coupling with the 1100-HPLC system (Agilent Technologies). 
The parameters of isolation and elution condition, ion monitor model and the molecular ion were all previously described in detail (16). The detailed information is as follows. The gradient elution of the mobile phase was as follows: $85 \%$ methanol (containing $15 \% \mathrm{H}_{2} \mathrm{O} ; \mathrm{pH} 7.5$ ) was kept for the first $3 \mathrm{~min}$, followed by a linear gradient from 85 to $100 \%$ methanol for $2 \mathrm{~min}$, and then $100 \%$ methanol was continued for another $15 \mathrm{~min}$. Finally, the elution condition went back to $85 \%$ methanol for $2 \mathrm{~min}$ at a flow rate of $0.7 \mathrm{ml} / \mathrm{min}$. Column temperature was kept at $40^{\circ} \mathrm{C}$. Ion detection was monitored by $\mathrm{APCI}^{+}$-MRM mode. The molecular ions were monitored at $m / z 348.00 / 62.00$ for AEA, $m / z$ 379.10/287.10 for 2-AG, $\mathrm{m} / \mathrm{z}$ 313.1/62.0 for C17:1 FAE, $m / z$ 464.4/264.2 for $\mathrm{C} 12: 0$ ceramide, $\mathrm{m} / \mathrm{z}$ 520.4/264.2 for C16:0 ceramide, $\mathrm{m} / \mathrm{z} 534.3 / 264.2$ for C17:0 ceramide, $m / z 548.4 / 264.2$ for $\mathrm{C} 18: 0$ ceramide, $m / z 576.4 / 264.2$ for C20:0 ceramide, $\mathrm{m} / \mathrm{z}$ 604.5/264.2 for C22:0 ceramide, $\mathrm{m} / \mathrm{z}$ 632.4/264.2 for C24:0 ceramide, and $\mathrm{m} / \mathrm{z} 630.4 / 264.2$ for C24:1 ceramide.

Enzymatic assays. Tissue samples were cut into $200 \mu \mathrm{m}$ thick sections and were homogenized in ice-cold Tris-HCl buffer (50 mM, pH 7.4) containing $0.32 \mathrm{M}$ sucrose to obtain total proteins. To measure FAAH and MAGL activity, tissue proteins were incubated at $37^{\circ} \mathrm{C}$ for $30 \mathrm{~min}$ in $50 \mathrm{mM}$ Tris- $\mathrm{HCl}$ buffer (pH 8.0, containing $0.05 \%$ fatty acid-free BSA), and $100 \mu \mathrm{g}$ sample protein was incubated with $50 \mu \mathrm{M}$ AEA or 2-oleoylglycerol as substrates. The reaction was stopped by adding $200 \mu \mathrm{l}$ chloroform/methanol (1:1, v/v), containing C17:0 heptadecanoic acid as an internal standard. The reaction solution was centrifuged at $1,500 \mathrm{x} \mathrm{g}$ at $4^{\circ} \mathrm{C}$ for $5 \mathrm{~min}$ and the organic layers were subsequently collected and dried under nitrogen. The residues were re-dissolved in $100 \mu \mathrm{l}$ methanol, and analyzed by 3200 Q Trap HPLC-MS system (Applied Biosystems; Thermo Fisher Scientific, Inc.) coupled with the 1100-HPLC system (Agilent Technologies, Inc.) in the negative-ion mode using 17:0 heptadecanoic acid as internal standard. A Hypersil Gold C18 column (dimensions, $250 \times 4.6 \mathrm{~mm}$; particle size, $5 \mu \mathrm{m}$; Thermo Fisher Scientific, Inc.) was used for analytical separation and the column temperature was kept at $40^{\circ} \mathrm{C}$. Fatty acids were eluted using a linear gradient from $90 \%$ phase $\mathrm{A}$ (methanol containing $0.25 \%$ acetic acid and $5 \mathrm{mM}$ ammonium acetate) to $100 \%$ phase $\mathrm{B}$ (water containing $0.25 \%$ acetic acid and $5 \mathrm{mM}$ ammonium acetate) in $2.5 \mathrm{~min}$ at a flow rate of $1.0 \mathrm{ml} / \mathrm{min}$. Capillary voltage was set at $-4 \mathrm{kV}$ and the fragmentor voltage was $120 \mathrm{~V}$. Nitrogen was used as drying gas at a flow rate of 13 liters $/ \mathrm{min}$ and a temperature of $350^{\circ} \mathrm{C}$. Nebulizer pressure is set at $60 \mathrm{psi}$. $[\mathrm{M}-\mathrm{H}]^{-}$ion was monitored in the selected-ion monitoring (SIM) mode $(\mathrm{m} / \mathrm{z}=303$ for arachidonic acid, $\mathrm{m} / \mathrm{z}=281$ for oleic acid, and $m / z=269$ for 17:0 heptadecanoic acid).

Statistical analysis. Data are expressed as the means \pm standard error of the mean. Experiments were performed in triplicate. Student's t-test was performed using GraphPad Prism (version 5.01; GraphPad Software, Inc.), and $\mathrm{P}<0.05$ was considered to indicate a statistically significant difference.

\section{Results}

Characteristics of 67 patients with HCC. Tumor tissues and adjacent non-tumor tissues were collected from patients
Table I. Clinical pathological parameters of 67 patients with hepatocellular carcinoma.

\begin{tabular}{lc}
\hline Parameter & Value, $\mathrm{n}(\%)$ \\
\hline Sex & \\
Male & $51(76.1)$ \\
Female & $16(23.9)$ \\
HBsAg & \\
Positive & $62(92.5)$ \\
Negative & $5(7.5)$ \\
AFP $(\mathrm{ng} / \mathrm{ml})$ before surgery & \\
$>25$ & $42(62.7)$ \\
$\leq 25$ & $25(37.3)$ \\
Tumor stage (TNM) (36) & \\
I or II & $47(70.1)$ \\
III & $20(29.9)$ \\
Grade & \\
High & $17(25.4)$ \\
Middle & $13(19.4)$ \\
Low & $37(55.2)$ \\
Tumor diameter $(\mathrm{cm})$ & \\
$>5$ & $22(32.8)$ \\
$\leq 5$ & $45(67.2)$ \\
\hline
\end{tabular}

with HCC who underwent surgical resection between 2015 and 2016. The detailed clinical parameters of the 67 patients are listed in Table I. The mean age of the patients was $52.6 \pm 11.3$ years (range, $18-76$ years). In total, $83.6 \%$ of the patients were male, consistent with prior reports that HCC is more prevalent in males compared to females. It was reported that estrogen might repress HCC growth via inhibiting alternative activation of tumor-associated macrophages (17). Of the 67 samples collected, 65 were HBV infections, with a ratio of $92.5 \%$. It was consistent with the fact that $>80 \%$ of patients with HCC were HBV carriers in Fujian Province. As there was an insufficient number of HCC samples without HBV infection in the current study, the effect of HBV on the expression of these endogenous lipids and its role in promoting HCC was not investigated. Approximately $10 \%$ of the patients were recorded to have varying degrees of liver damage, and 23 patients had cancerous thrombi. In the cohort, $\sim 55 \%$ was diagnosed with intermediate-grade cancer, while the percentage of high- grade was $25.4 \%$ and that of low-grade cases was $19.4 \%$ according to the Standardization of diagnosis and treatment for hepatocellular carcinoma (2017 edition, Bureau of Medical Administration, National Health and Family Planning Commission of the PRC) (18).

According to the current data, 38 patients $(56 \%)$ have a recorded pathological status of cirrhosis, and 14 patients of them had Child-Pugh score. A total of 9 patients were recorded as HBV-related decompensated liver cirrhosis, 7 of them had a higher Child-Pugh score $(10.29 \pm 0.68)$, while 2 of them had a lower Child-Pugh score (5 and 6, respectively). A total of 4 patients were recorded as decompensated alcoholic cirrhosis with Child-Pugh scores of 5, 9, 12, and 13, respectively. And 
A
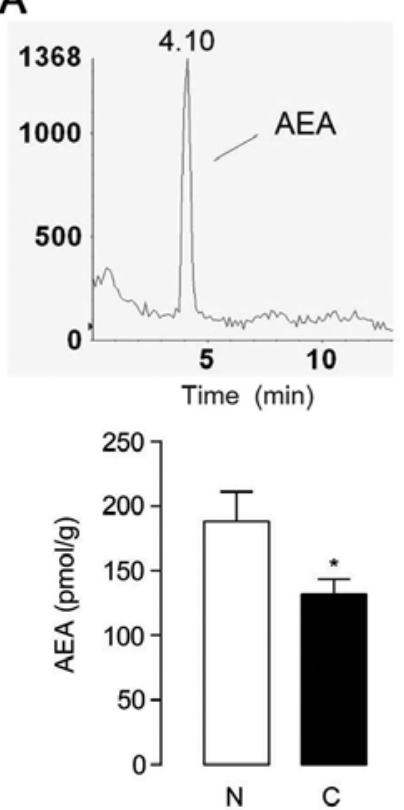

B
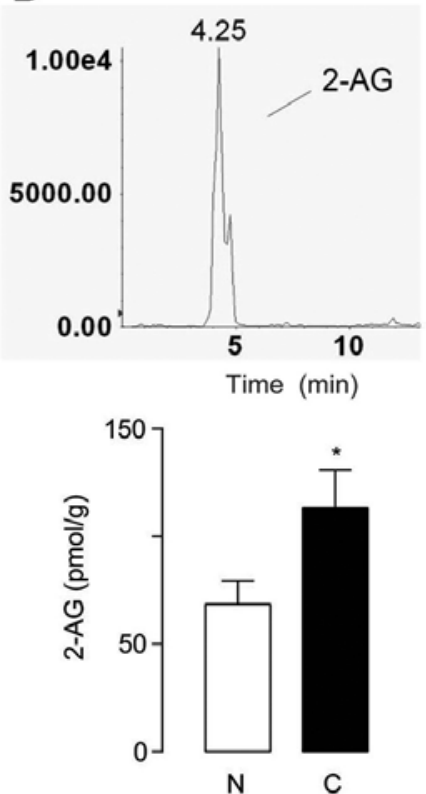

C
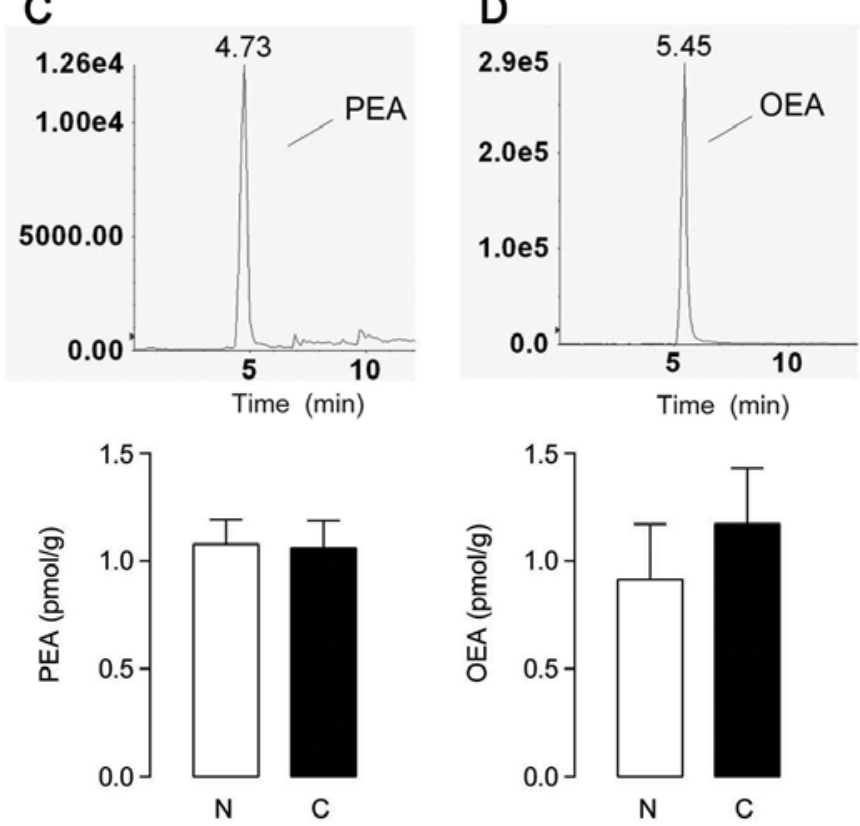

Figure 1. High-performance liquid chromatography-mass spectrometry analysis for endocannabinoids. Expression levels of (A) AEA, (B) 2-AG, (C) PEA, and (D) OEA in non-tumor tissue control and hepatocellular carcinoma tissues. ${ }^{*} \mathrm{P}<0.05 ; \mathrm{n}=67$. AEA, anandamide; 2-AG, 2-arachidonylglycerol; PEA, palmitoylethanolamide; OEA, oleoylethanolamide; N, non-tumor tissue control; $\mathrm{C}$, hepatocellular carcinoma tissues.

only one patient was recorded as cardiogenic cirrhosis with a Child-Pugh score of 5.

The present study also included 7 patients who were HCV RNA positive and 6 patients were HCV RNA negative. There are no relevant records for the other patients. Some patients showed dyslipidemia, 5 of them were diagnosed with fatty liver and hypertriglyceridemia, 3 patients were diagnosed with hypercholesterolemia, and only 1 patient was suspected as nonalcoholic steatohepatitis.

Expression of endocannabinoids in human HCC samples. The endogenous lipids in 67 tumor and matched non-cancerous tissue pairs were detected and quantified by HPLC-MS. Compared with the non-tumor counterparts, AEA levels were significantly decreased in tumor tissues $(\mathrm{P}=0.0329 ; 188.3 \pm 22.94 \mathrm{pmol} / \mathrm{g}$ in non-tumor tissues, and $131.7 \pm 12.04 \mathrm{pmol} / \mathrm{g}$ in HCC, Fig. 1A), while the level of 2-AG, another important endocannabinoid, was significantly increased in tumor tissues $(\mathrm{P}=0.0278 ; 68.55 \pm 10.64 \mathrm{nmol} / \mathrm{g}$ in non-cancerous controls, and $113.3 \pm 17.48 \mathrm{nmol} / \mathrm{g}$ in $\mathrm{HCC}$, Fig. 1B). As congeners of AEA, palmitoylethanolamide (PEA) and oleoylethanolamide (OEA) belong to the superfamily of $\mathrm{N}$-acylethnolamines (NAEs), however PEA and OEA interact with the PPAR- $\alpha$ receptor instead of cannabinoid receptors (19). The expression of PEA and OEA did not differ significantly between the two groups (Fig. 1C and D).

Changes in endocannabinoid metabolism in human HCC samples. The most notable endocannabinoid changes in the present study were the decrease of AEA and increase of 2-AG. AEA is generated by NAPE-PLD and metabolized by FAAH (20). The checkpoint in 2-AG synthesis is considered to be DGL- $\alpha$ (21). Furthermore, MAGL is mainly involved in 2-AG degradation (21). To further investigate the molecular mechanisms underlying the aforementioned changes, the expression and activity profiles of these enzymes were examined in tissue samples.

There was no significant difference in the mRNA expression of NAPE-PLD between tumors and non-cancerous controls (Fig. 2A). The mRNA levels and activity of FAAH increased in HCC samples relative to their non-tumor counterparts $(\mathrm{P}=0.041$; Fig. 2B and C), which suggested increased degradation of AEA in tumor tissues. A 5-fold higher level of DGL- $\alpha$ mRNA was detected in the tumor group $(1.05 \pm 0.09$ in non-tumor tissues and 5.27 \pm 0.81 in HCC; $\mathrm{P}<0.0001$; Fig. 2D). The expression of DGL- $\alpha$ exhibited a greater increase compared with MAGL (Fig. 2E and F), indicating that 2-AG synthesis was markedly faster than its degradation. Whether the elevated expression of 2-AG and DGL- $\alpha$ may be used as markers for early HCC diagnosis warrants further investigation.

Expression of cannabinoid receptors in human HCC samples. It has been reported that cannabinoid receptors 1 and 2 mediate functional responses to the AEA and 2-AG. As the levels of these two endocannabinoids changed significantly in tumor tissues, the present study investigated whether there were corresponding changes in the receptors. The mRNA expression of $\mathrm{CB}_{1}$ and $\mathrm{CB}_{2}$ was examined by quantitative PCR in both tumor and non-tumor samples. There was no significant difference between the two groups for $\mathrm{CB}_{1}$ mRNA levels (Fig. 3A), while $\mathrm{CB}_{2}$ expression was increased in tumor tissues (Fig. 3C). The protein levels of the two receptors were further investigated via western blot analysis. ImageJ software was used to calculate the optical density of the blots, using $\beta$-actin as the control. The results revealed that $\mathrm{CB}_{1}$ protein levels in tumor tissues were decreased significantly $(1.45 \pm 0.054$ in non-cancerous tissues and $0.74 \pm 0.086$ in tumor tissues; $P=0.0022$; Fig. $3 \mathrm{~B}$ ), and those of $\mathrm{CB}_{2}$ were significantly increased $(1.37 \pm 0.088$ in non-tumor 
A
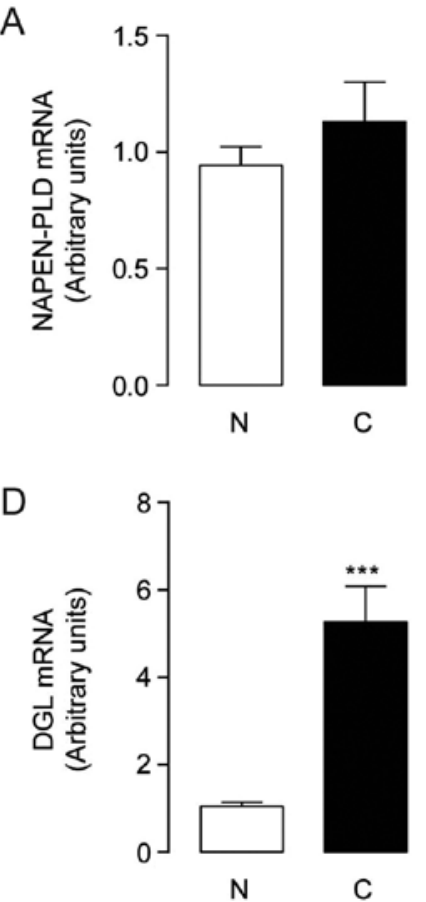

B

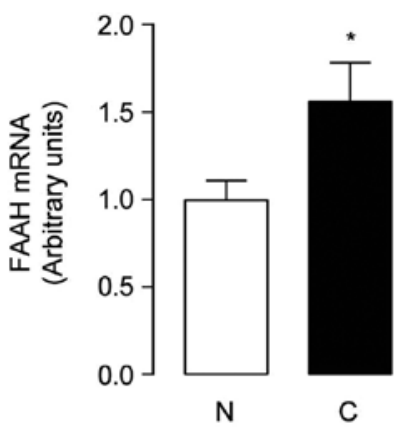

E

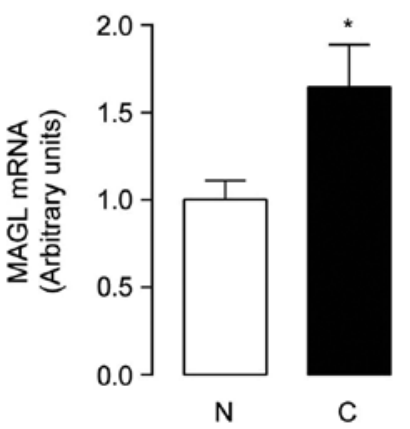

C

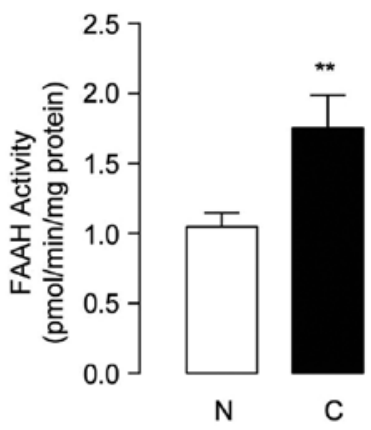

$\mathrm{F}$

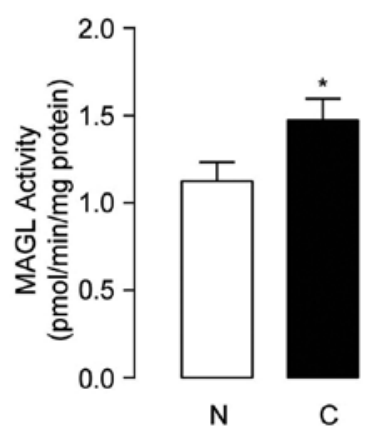

Figure 2. Alteration of endocannabinoids metabolic enzymes in human hepatocellular carcinoma. The mRNA expression levels (A, B, D, E) and the enzyme activities (C and F) of (A) NAPE-PLD, (B and C)FAAH,(D) DGL and (E and F) MAGL in non-tumor tissue control and hepatocellular carcinoma tissues. ${ }^{*} \mathrm{P}<0.05,{ }^{* * *} \mathrm{P}<0.01$ and ${ }^{* * * *} \mathrm{P}<0.001 ; \mathrm{n}=67 . \mathrm{N}$, non-tumor tissue control; $\mathrm{C}$, hepatocellular carcinoma tissues; NAPE-PLD, $N$-acylphosphatidylethanolamine-hydrolysingphospholipase D; FAAH, fatty acid amide hydrolase; MAGL, monoacylglycerol lipase; DGL, diacylglycerol lipase.

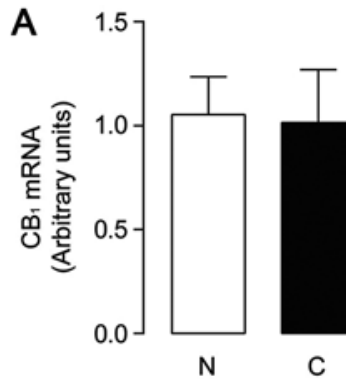

C

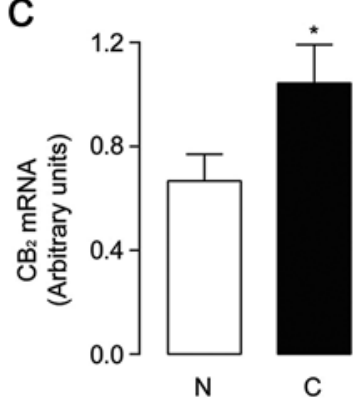

B

$\mathrm{CB}_{1}$

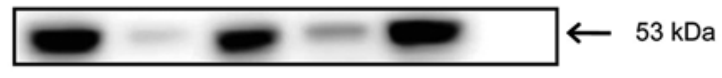

$\beta$-actin
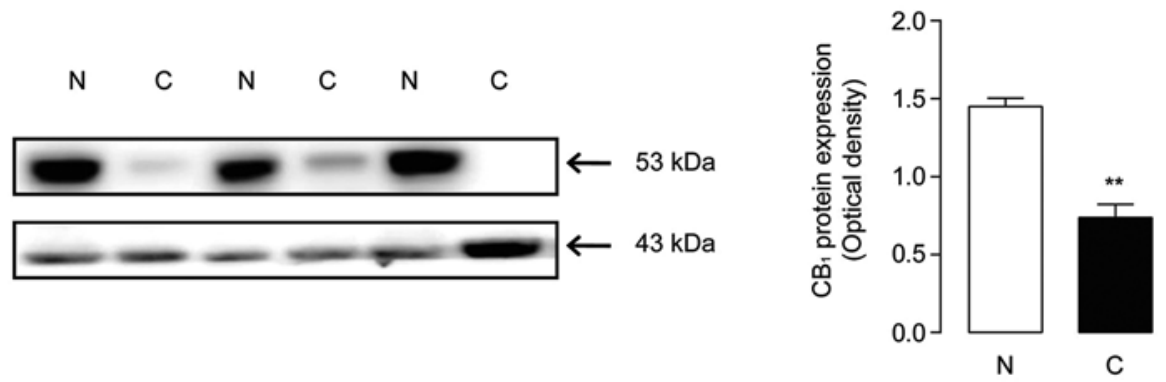

D

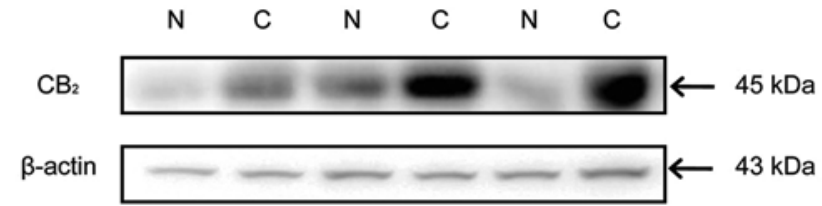

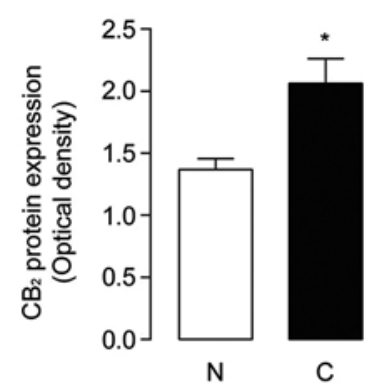

Figure 3. Expression levels of cannabinoid receptors 1 and 2 in human hepatocellular carcinoma. The mRNA expression levels (A and C) and the protein levels $(B$ and $D)$ of $(A$ and $B) C_{1}$ receptor and $(C$ and $D) C_{2}$ receptor in non-tumor tissue control and hepatocellular carcinoma tissues. " $P<0.05$ and ${ }^{* * *} \mathrm{P}<0.01$; $\mathrm{n}=67$. $\mathrm{N}$, non-tumor tissue control; $\mathrm{C}$, hepatocellular carcinoma tissues; $\mathrm{CB}$, cannabinoid receptor.

tissues and $2.06 \pm 0.2$ in tumor tissues; $\mathrm{P}=0.0336$, Fig. 3D). The trends in receptor protein expression were consistent with the changes in endogenous ligands.

Expression of endogenous ceramides in human HCC samples. Ceramides are a family of bioactive sphingolipids with tumor-suppressive properties (22). In the present study, HPLC-MS detection revealed that endogenous C12:0, C16:0, C18:1 and C24:1-ceramides were markedly increased in HCC tissues, whereas the levels of C18:0,C20:0 and C24:0-ceramides were significantly decreased in these samples, each compared with their adjacent normal counterparts (Fig. 4). The 

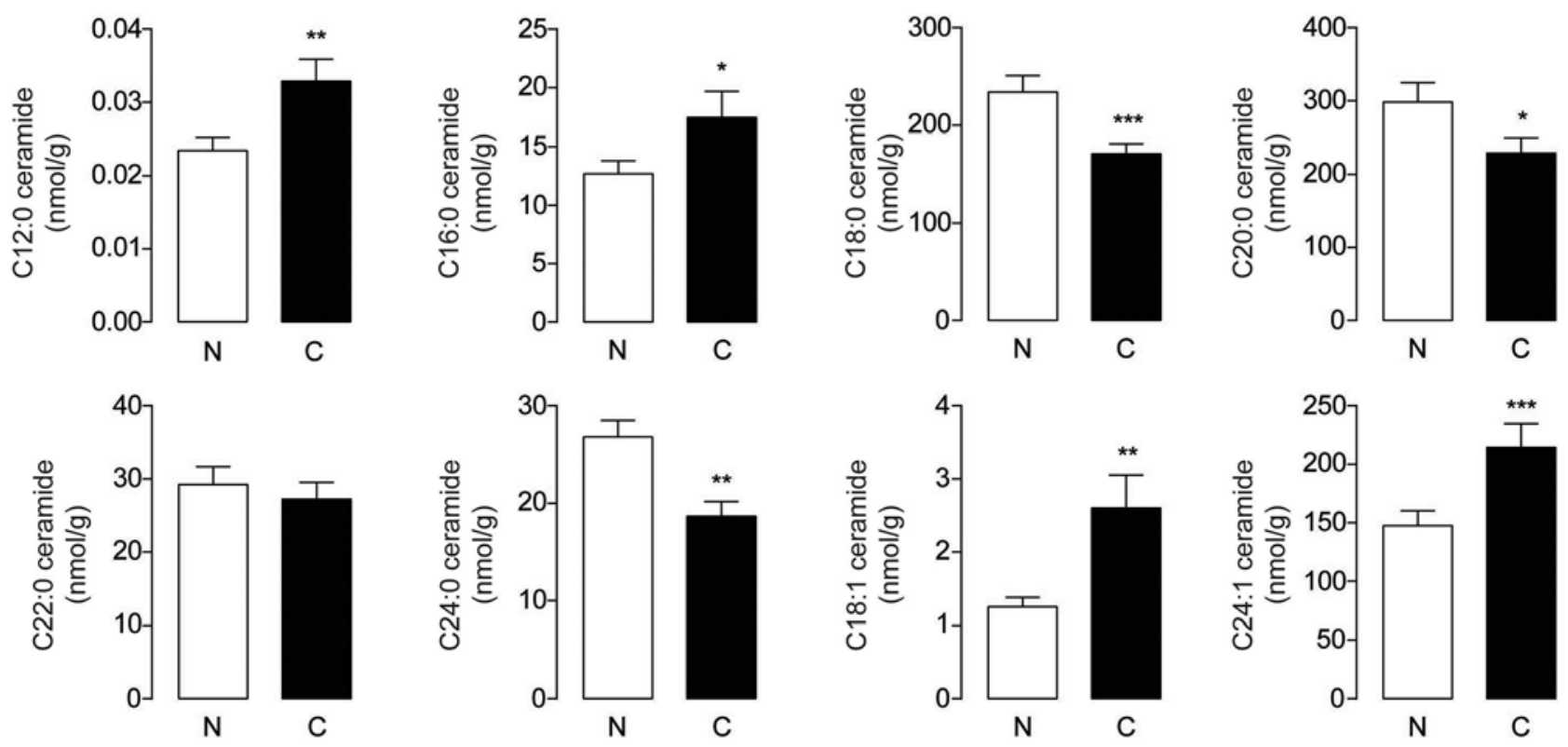

Figure 4. Expression levels of endogenous ceramides in non-tumor tissue control and hepatocellular carcinoma tissues. ${ }^{*} \mathrm{P}<0.05,{ }^{* * *} \mathrm{P}<0.01$ and ${ }^{* * *} \mathrm{P}<0.001 ; \mathrm{n}=67$. $\mathrm{N}$, non-tumor tissue control; $\mathrm{C}$, hepatocellular carcinoma tissues.

differences in C22:0-ceramide levels between tumor and non-tumor tissues were not significant. It was documented that C18:0-ceramide exerted anti-proliferative effects on head and neck squamous cell carcinomas (HNSCCs), while the roles of C18:0, C20:0 and C24:0-ceramides in the pathogenesis and progression of HCC require further investigation.

Changes in endogenous ceramide metabolism in human HCC samples. To examine the mechanisms underlying the changes observed with ceramides, the mRNA levels of CerS1-6 were examined in 67 pairs of HCC tumor and adjacent non-tumor tissues (Fig. 5A-F). The results revealed that the mRNA levels of CerS6 increased significantly in $\sim 70 \%$ of the patients, which were associated with increased tumor levels of C16:0-ceramide. Furthermore, the decreased mRNA levels of CerS1 were in accordance with reduced C18:0-ceramide levels, which was highly relevant to the lower levels of C18:0-ceramide observed in HCC tissues. Decreases in C20:0 and C24:0-ceramides in tumor tissues may be attributed to the downregulated expression of CerS4, a synthase responsible for the synthesis of C18:0-24:0 ceramides (22). Notably, the levels of mono-unsaturated C18:1 and C24:1 ceramides were increased in tumor samples, consistently with the elevated levels of dihydroceramide desaturase 1 and 2 (DEGS-1/2) (Fig. 5G and H). However, the main role of DEGS and mono-unsaturated ceramides involved in HCC formation and progression warrants further investigation.

SCD1 is a transmembrane protein that converts SFAs to $\Delta-9$ MUFAs to supply phospholipids for membrane biogenesis during cancer cell proliferation (23). The SCD1 mRNA and protein levels were significantly upregulated in HCC tissues compared with their non-tumor counterparts (Fig. 5I). High expression of SCD1 may be associated with the suppression of C18:0, 20:0 and 24:0-ceramide de novo synthesis, while the crosstalk between the SCD1 pathway and the ceramide metabolism network has, to the best of our knowledge, yet to be investigated.

\section{Discussion}

It has been reported that endocannabinoids are generally upregulated in tumor tissues, including gliomas, colon and breast cancer, compared with non-cancerous tissues. AEA is the first discovered endogenous ligand for cannabinoid receptor 1. Administration of AEA or inhibitors of FAAH, the main degradative enzyme of AEA, has been shown to suppress the growth of different types of tumor xenografts via $\mathrm{CB}$ receptor activation (24). Targeting endocannabinoid systems may be a reasonable strategy for anticancer therapy. In previous studies, endocannabinoids and the associated receptors were detected in tissues and serum of patients with HCC by lipid analysis, qPCR, and immunohistochemistry. However, a disadvantage of the aforementioned is that there is no systematic detection of the endocannabinoid system, as they cannot explain the reason for these major endocannabinoids levels changes. The novelty of this research is that a systematic detection including endocannabinoids, ceramides, the associated synthetic and metabolic hydrolases and the associated receptors is carried out. The changes of endogenous lipids have been explained primarily to provide potential targets and research directions for the diagnosis and treatment of HCC.

To date, to the best of our knowledge, little is known on the physiological and pathological roles of AEA in HCC. In a serum metabolic profiling analysis study of $\mathrm{HCC}$, Zhou et al (25) found that serum AEA was significantly elevated in HCC groups compared with healthy controls. In the present study, endogenous AEA levels were decreased in liver cancer tissues, contrary to a previous report (25). The downregulation of AEA in HCC tissues reported herein was consistent with this study's findings that the expression and activity of FAAH increased significantly in HCC. In the 
A

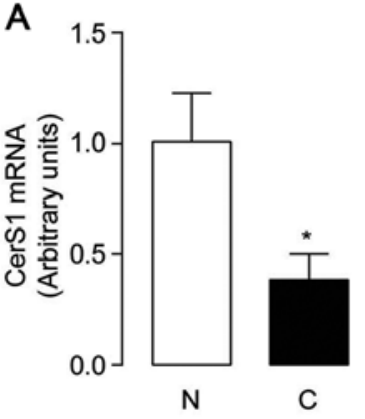

E

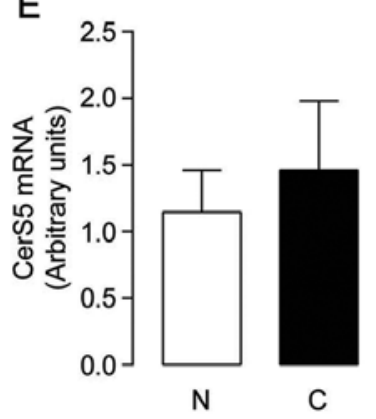

B

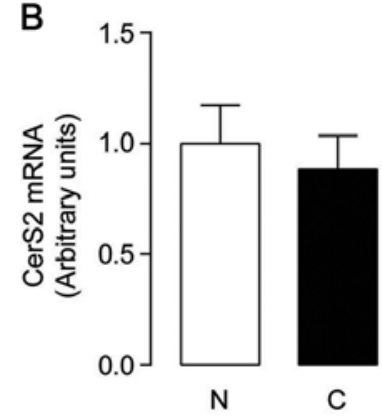

F

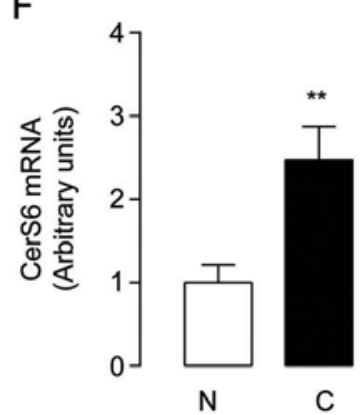

C

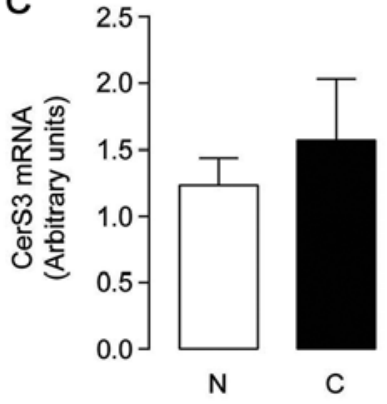

G

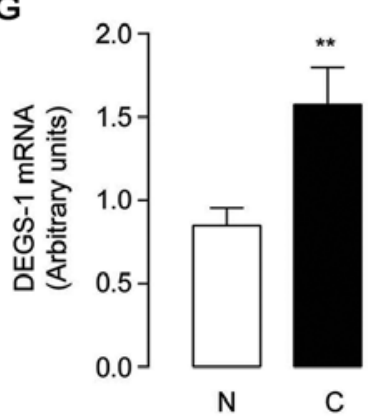

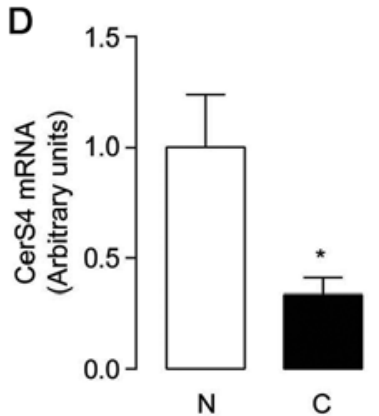

$\mathrm{H}$

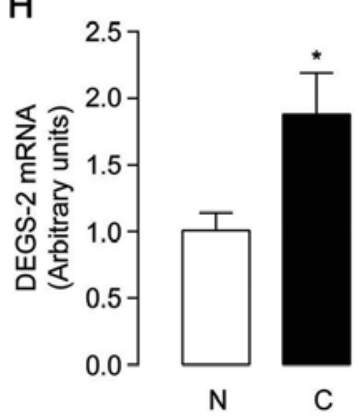

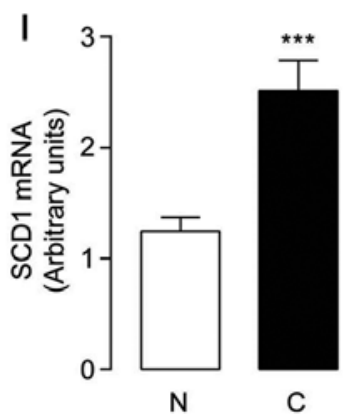

J

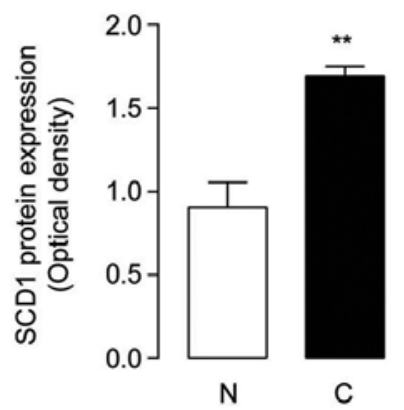

Figure 5. The mRNA expression levels of (A-F) CerS1-6, (G and H) DEGS1-2, (I) SCD1 and protein level of (J) SCD1 in non-tumor tissue control and hepatocellular carcinoma tissues. ${ }^{*} \mathrm{P}<0.05,{ }^{* * *} \mathrm{P}<0.01$ and ${ }^{* * * *} \mathrm{P}<0.001, \mathrm{n}=67$. CER, ceramide synthases; $\mathrm{N}$, non-tumor tissue control; $\mathrm{C}$, hepatocellular carcinoma tissues; DEGS-1/2, dihydroceramide desaturase 1 and 2; SCD1, stearoyl-CoA desaturase.

study conducted by Xu et al (26), serum was used instead of liver tissues, recapitulating the systematic AEA metabolic profiles of patients with HCC. This study only revealed the metabolic characteristics of AEA in the local environment of the liver. Therefore, this study suggests that a decrease in AEA levels in tumor tissues shows that AEA may play an important role in suppressing $\mathrm{HCC}$ cell proliferation. AEA is an endogenous agonist of the $\mathrm{CB}_{1}$ receptor, and the activation of $\mathrm{CB}_{1}$ is beneficial for tumor cell apoptosis and autophagy. After treatment with cannabinoids, the expression levels of Akt in breast cancer cells decreased and the PI3K/Akt/mTOR pathway was inhibited, which significantly suppressed tumor cell migration. Elevated serum AEA levels may reflect antitumor auto-protection of the body; however, the precise role of endogenous AEA in the occurrence and progression of cancer remains unclear.

$\mathrm{CB}_{1}$ receptors are preferentially expressed in the central nervous system. In the present study, a notable decrease of $\mathrm{CB}_{1}$ receptor expression in $\mathrm{HCC}$ tissues was observed by western blot analysis. However, it has been reported that high expression of the $\mathrm{CB}_{1}$ receptor was observed in $45 \%$ of the cases of HCC by immunohistochemical analysis (26). These conflicting results may be attributed to the selection of different differentiation samples. The data demonstrated that high $\mathrm{CB}_{1}$ expression was associated with high differentiation of HCC (26), however poorly differentiated HCCs generally exhibited low $\mathrm{CB}_{1}$ expression. In the present study, $74.6 \%$ of $\mathrm{HCC}$ samples were poorly to intermediately differentiated, with lower $\mathrm{CB}_{1}$ levels, in accordance with the aforementioned data, and $\mathrm{CB}_{1}$ receptor expression in $\mathrm{HCC}$ was suggested to be associated with improved prognosis (26).

It was recently reported that the $\mathrm{CB}_{1}$ receptor was upregulated in diethynitrosamine-induced HCC mouse models (27). Peripheral blockade or genetic ablation of the $\mathrm{CB}_{1}$ receptor suppressed its high expression in the endocannabinoid system and the growth of HCC. However, these conclusions were drawn from observations made with an animal model; therefore, they cannot fully represent the true conditions of the human body. Accordingly, further research is required to validate that the pharmacological activation of $\mathrm{CB}_{1}$ by enhancing AEA levels is effective for the treatment of HCC. 
The most distinct change to the endocannabinoid system in the present study was the increase in 2-AG. The activity and expression of MAGL, the enzyme responsible for 2-AG hydrolysis, was increased in HCC compared with non-cancerous controls, which was not consistent with the increase of 2-AG. However, the expression of DGL- $\alpha$, the 2-AG-biosynthesizing enzyme, increased in HCC tissues, suggesting faster biosynthesis of 2-AGcompared with its degradation. The upregulation of MAGL may be a result of the negative feedback induced by the excessive production of DGL- $\alpha$ and 2-AG.

It has been reported that $\mathrm{CB}_{2}$ receptors are more prevalent in peripheral tissues associated with immune function. Hepatic $\mathrm{CB}_{2}$ protein levels are elevated in a number of liver pathologies, including fatty liver, hepatic fibrogenesis (28) and acute liver injury (29). In 2006, by immunohistochemical analysis, $\mathrm{Xu}$ et al (26) showed high expression of $\mathrm{CB}_{1}$ and $\mathrm{CB}_{2}$ receptors in 45 and $52 \%$ cases of $\mathrm{HCC}$, respectively, which is consistent with this study's findings. Overexpression of $\mathrm{CB}_{2}$ may have potential as prognostic indicators for patients with HCC.

Studies have shown that the major endocannabinoid 2-AG and the associated receptor $\mathrm{CB}_{2}$ have a key role in the pathogenesis of chronic liver injury. Activation of $\mathrm{CB}_{2}$ receptor is known to inhibit tumor vascularization (30), while, in another research, elevated $\mathrm{CB}_{2}$ level was reported to facilitate the tumor invasion through the suppression of the anti-tumor immune system (31). The detailed role of the $\mathrm{CB}_{2}$ receptor in HCC development and progression remains unknown. To the best of our knowledge, there are currently no reports on $\mathrm{CB}_{2}$ function in the treatment of HCC.

The present study provided new evidence confirming the excessive expression of $2-\mathrm{AG}$ and its associated receptor $\mathrm{CB}_{2}$ in individuals with $\mathrm{HCC}$, which may lead to the identification of novel diagnostic and therapeutic targets against HCC. However, there was no significant difference for 2-AG and $\mathrm{CB}_{2}$ expression between stage I-II and III, or between Grade high, middle, and low of HCC. Therefore, further studies should delineate whether administration of $\mathrm{CB}_{2}$ agonist/antagonist may pave the way for anti-HCC treatment.

The expression levels of major endocannabinoids, AEA and 2-AG, are modulated mainly through synthetic and metabolic enzymes. However, changes in endocannabinoids levels cannot be accurately explained only through the above enzymes. The aforementioned suggests that by inhibiting highly expressed metabolic enzymes, local endocannabinoid levels can be increased, which seems likely to have some effects on tumor cells through activating $\mathrm{CB}$ receptors. It was reported that the inhibitors of FAAH and MGL, the metabolic enzymes of AEA and 2-AG, showed anti-tumor effects in some types of malignancies (21), including colorectal cancer (9), non-small lung cancer (32)and prostate cancer (33). However, the precise role of endocannabinoids system in the occurrence and progression of HCC is still unclear. In the present research, of the 67 patients with $\mathrm{HCC}$, only 5 patients who were HBV negative were included. The proportion of patients with hepatitis $B$ positive reached $92.5 \%$. Future studies including a sufficient sample of patients with $\mathrm{HCC}$ and hepatitis B negative should be conducted to provide the metabolic profiling data for $\mathrm{HBV}$ infection.

Cannabinoid receptor activation was shown to induce apoptosis via de novo ceramide synthesis in several types of cancer cells, including glioma (5) and colon cancer cells (34). Ceramides are a class of pro-apoptotic sphingolipids with important roles in the control of cancer cell fate. It has been reported that the levels of specific ceramides, such as C18:0 and C20:0, may be important in the inhibition of cell growth in human HNSCC (35). In the present study, notable decreases in C18:0, 20:0 and 24:0-ceramides were found in HCC tissues compared with non-tumor counterparts, which was in accordance with previously reported data (35). In accordance with these findings in the present study, the levels of C12:0, C16:0, C18:1 and C24:1-ceramides were increased in HCC tumors compared with adjacent normal tissues. The specific role of the changes in endogenous ceramide expression in HCC has not been fully elucidated, and further studies are required to determine the possible association between altered ceramide levels and HCC progression. Increasing ceramide levels is a potential strategy for the treatment of HCC, and the inhibition of SCD1 in HCC may be a viable option (10).

\section{Acknowledgements}

The authors would like to thank Dr Xuan Zhu (School of Pharmaceutical Sciences, Xiamen University) for their help in revising the manuscript.

\section{Funding}

The present study was supported by the National Natural Science Foundation of China (no. 81603145), the Fujian Health-Education Research Grant (no. WKJ2016-2-03), Fujian Provincial Natural Science Foundation (nos. 2015J01416 and 2017J01146), and the Xiamen Science and Technology Program Grant (no. 3502Z20154069).

\section{Availability of data and materials}

The datasets used and/or analyzed during the present study are available from the corresponding author on reasonable request.

\section{Authors' contributions}

JY and FQ conceived and designed experiments. JY and LL carried out the experiments. RZ analyzed data, prepared figures, and finished the complementary experiments. JY, FQ and RZ wrote the manuscript. YT, JY and FQ provided the clinical samples. All the authors have read and approved the final version of this manuscript.

\section{Ethics approval and consent to participate}

The present study was approved by The Ethics Committee of the Fifth Hospital of Xiamen (Fuzhou, China) following the clinical registration guidelines in China. All patients provided written informed consent for the investigation.

\section{Patient consent for publication}

All patients consented to the publication of this research. 


\section{Competing interests}

The authors declare that they have no competing interests.

\section{References}

1. Allemani C, Matsuda T, Di Carlo V, Harewood R, Matz M, Nikšić M, Bonaventure A, Valkov M, Johnson CJ, Estève J, et al Global surveillance of trends in cancer survival 2000-14 (CONCORD-3): Analysis of individual records for 37513025 patients diagnosed with one of 18 cancers from 322 populationbased registries in 71 countries. Lancet 391: 1023-1075, 2018.

2. Strickaert A, Corbet C, Spinette SA, Craciun L, Dom G, Andry G, Larsimont D, Wattiez R, Dumont JE, Feron O and Maenhaut C: Reprogramming of energetic metabolism: Increased expression and roles of pyruvate carboxylase in papillary thyroid cancer. Thyroid, 2019 (Epub ahead of print).

3. Zhou X, Yang X, Sun X, Xu X, Li X, Guo Y, Wang J, Li X, Yao L, Wang $\mathrm{H}$ and Shen L: Effect of PTEN loss on metabolic reprogramming in prostate cancer cells. Oncol Lett 17: 2856-2866, 2019.

4. Li X, Xu H, Lei T, Yang Y, Jing D, Dai S, Luo P and Xu Q: A pulsed electromagnetic field protects against glutamate-induced excitotoxicity by modulating the endocannabinoid system in HT22 cells. Front Neurosci 11: 42, 2017.

5. Dyall SC, Mandhair HK, Fincham REA, Kerr DM, Roche M and Molina-Holgado F: Distinctive effects of eicosapentaenoic and docosahexaenoic acids in regulating neural stem cell fate are mediated via endocannabinoid signalling pathways. Neuropharmacology 107: 387-395, 2016.

6. Cinar R, Godlewski G, Liu J, Tam J, Jourdan T, Mukhopadhyay B, Harvey-White J and Kunos G: Hepatic cannabinoid-1 receptors mediate diet-induced insulin resistance by increasingde novosynthesis of long-chain ceramides. Hepatology 59: 143-153, 2014.

7. Sean M. Emery AHL and David A: Gewirtz: Involvement of the endocannabinoid system in the development and treatment of breast cancer. Virginia commonwealth univ richmond, 2014.

8. Orellana-Serradell O, Poblete CE, Sanchez C, Castellón EA, Gallegos I, Huidobro C, Llanos MN and Contreras HR: Proapoptotic effect of endocannabinoids in prostate cancer cells. Oncol Rep 33: 1599-1608, 2015.

9. Ligresti A, Bisogno T, Matias I, De Petrocellis L, Cascio MG Cosenza V, D'argenio G, Scaglione G, Bifulco M, Sorrentini I and Di Marzo V: Possible endocannabinoid control of colorectal cancer growth. Gastroenterology 125: 677-687, 2003.

10. Galve-Roperh I, Sánchez C, Cortés ML, Gómez del Pulgar T, Izquierdo $\mathrm{M}$ and Guzmán M: Anti-tumoral action of cannabinoids: Involvement of sustained ceramide accumulation and extracellular signal-regulated kinase activation. Nat Med 6: 313-319, 2000.

11. Igal RA: Stearoyl CoA desaturase-1: New insights into a central regulator of cancer metabolism. Biochim Biophys Acta 12: 1865-1880, 2016.

12. Chen L, Ren J, Yang L, Li Y, Fu J, Li Y, Tian Y, Qiu F, Liu Z and Qiu Y: Stearoyl-CoA desaturase-1 mediated cell apoptosis in colorectal cancer by promoting ceramide synthesis. Sci Rep 6: 19665,2016

13. Li X, Wenes M, Romero P, Huang SC, Fendt SM and Ho PC: Navigating metabolic pathways to enhance antitumour immunity and immunotherapy. Nat Rev Clin Oncol, 2019 (Epub ahead of print)

14. Mittal S and El-Serag HB: Epidemiology of HCC: consider the population. J Clin Gastroenterol 47: S2-S6, 2013.

15. Livak KJ and Schmittgen TD: Analysis of relative gene expression data using real-time quantitative PCR and the 2(-Delta Delta C(T)) method. Methods 25: 402-408, 2001

16. Chen L, Chen H, Li Y, Li L, Qiu Y and Ren J: Endocannabinoid and ceramide levels are altered in patients with colorectal cancer. Oncol Rep 34: 447-454, 2015.

17. Yang W, Lu Y, Xu Y, Xu L, Zheng W, Wu Y, Li L and Shen P: Estrogen represses hepatocellular carcinoma (HCC) growth via inhibiting alternative activation of tumor-associated macrophages (TAMs). J Biol Chem 287: 40140-40149, 2012.

18. Zhou J, Sun HC, Wang Z, Cong WM, Wang JH, Zeng MS, Yang JM, Bie P, Liu LX, Wen TF, et al: Guidelines for diagnosis and treatment of primary liver cancer in China (2017 Edition). Liver Cancer 7: 235-260, 2018
19. Fu J, Gaetani S, Oveisi F, Lo Verme J, Serrano A, Rodríguez De Fonseca F, Rosengarth A, Luecke H, Di Giacomo B, Tarzia G and Piomelli D: Oleylethanolamide regulates feeding and body weight through activation of the nuclear receptor PPAR-alpha. Nature 425: 90-93, 2003.

20. Bobrov MY, Shevchenko VP, Yudushkin IA, Rogov SI, Remov MN, Fomina-Ageeva EV, Gretskaya NM, Nagaev IY, Kuklev DV and Bezuglov VV: Hydrolysis of anandamide and eicosapentaenoic acid ethanolamide in mouse splenocytes. Biochemistry 65: 615-619, 2000.

21. Nomura DK, Long JZ, Niessen S, Hoover HS, Ng SW and Cravatt BF: Monoacylglycerol lipase regulates a fatty acid network that promotes cancer pathogenesis. Cell 140: 49-61, 2010.

22. Morad SA and Cabot MC: Ceramide-orchestrated signalling in cancer cells. Nat Rev Cancer 13: 51-65, 2013.

23. Matsui H, Yokoyama T, Sekiguchi K, Iijima D, Sunaga H, Maniwa M, Ueno $M$, Iso $T$, Arai $M$ and Kurabayashi $M$ : Stearoyl-CoA desaturase-1 (SCD1) augments saturated fatty acid-induced lipid accumulation and inhibits apoptosis in cardiac myocytes. PLoS One 7: e33283, 2012.

24. Massi P, Valenti M, Vaccani A, Gasperi V, Perletti G, Marras E, Fezza F, Maccarrone M and Parolaro D: 5-Lipoxygenase and anandamide hydrolase (FAAH) mediate the antitumor activity of cannabidiol, a non-psychoactive cannabinoid. J Neurochem 104: 1091-1100, 2008

25. Zhou L, Ding L, Yin P, Lu X, Wang X, Niu J, Gao P and Xu G: Serum metabolic profiling study of hepatocellular carcinoma infected with hepatitis B or hepatitis C virus by using liquid chromatography-mass spectrometry. J Proteome Res 11: 5433-5442, 2012

26. Xu X, Liu Y, Huang S, Liu G, Xie C, Zhou J, Fan W, Li Q, Wang Q, Zhong D and Miao X: Overexpression of cannabinoid receptors CB1 and CB2 correlates with improved prognosis of patients with hepatocellular carcinoma. Cancer Genet Cytogenet 171: 31-38, 2006.

27. Mukhopadhyay B, Schuebel K, Mukhopadhyay P, Cinar R, Godlewski G, Xiong K, Mackie K, Lizak M, Yuan Q, Goldman D and Kunos G: Cannabinoid receptor 1 promotes hepatocellular carcinoma initiation and progression through multiple mechanisms. Hepatology 61: 1615-1626, 2015.

28. Louvet A, Teixeira-Clerc F, Chobert MN, Deveaux V, Pavoine C, Zimmer A, Pecker F, Mallat A and Lotersztajn S: Cannabinoid CB2 receptors protect against alcoholic liver disease by regulating Kupffer cell polarization in mice. Hepatology 54: 1217-1226, 2011

29. Teixeira-Clerc F, Belot MP, Manin S, Deveaux V, Cadoudal T, Chobert MN, Louvet A, Zimmer A, Tordjmann T, Mallat A and Lotersztajn S: Beneficial paracrine effects of cannabinoid receptor 2 on liver injury and regeneration. Hepatology 52: 1046-1059, 2010

30. Blázquez C, Casanova ML, Planas A, Gómez Del Pulgar T, Villanueva C, Fernández-Aceñero MJ, Aragonés J, Huffman JW, Jorcano JL and Guzmán M: Inhibition of tumor angiogenesis by cannabinoids. FASEB J 17: 529-531, 2003.

31. Hermanson DJ and Marnett LJ: Cannabinoids, endocannabinoids, and cancer. Cancer Metast Rev 30: 599-612, 2011.

32. Ravi J, Sneh A, Shilo K, Nasser MW, Nasser MW and Ganju RK: FAAH inhibition enhances anandamide mediated anti-tumorigenic effects in non-small cell lung cancer by downregulating the EGF/EGFR pathway. Oncotarget 5: 2475-2486, 2014.

33. Nomura DK, Lombardi DP, Chang JW, Niessen S, Ward AM, Long JZ, Hoover HH and Cravatt BF: Monoacylglycerol lipase exerts dual control over endocannabinoid and fatty acid pathways to support prostate cancer. Chem Biol 18: 846-856, 2011.

34. Cianchi F, Papucci L, Schiavone N, Lulli M, Magnelli L, Vinci MC, Messerini L, Manera C, Ronconi E, Romagnani P, et al: Cannabinoid receptor activation induces apoptosis through tumor necrosis factor $\alpha$-mediated ceramide de novo synthesis in colon cancer cells. Clin Cancer Res 14: 7691-7700, 2008.

35. Karahatay S, Thomas K, Koybasi S, Senkal CE, Elojeimy S, Liu X, Bielawski J, Day TA, Gillespie MB, Sinha D, et al: Clinical relevance of ceramide metabolism in the pathogenesis of human head and neck squamous cell carcinoma (HNSCC): Attenuation of C(18)-ceramide in HNSCC tumors correlates with lymphovascular invasion and nodal metastasis. Cancer Lett 256: 101-111, 2007.

36. Befeler AS and Di Bisceglie AM: Hepatocellular carcinoma: Diagnosis and treatment. Gastroenterology 122: 1609-1619, 2002. 